\title{
Lessons to be learnt from other countries about mandatory child vaccination
}

\author{
Denes Stefler PhD student in cardiovascular epidemiology ${ }^{1}$, Raj Bhopal Bruce and John Usher \\ professor of public health ${ }^{2}$
}

${ }^{1}$ Department of Epidemiology and Public Health, University College London, London WC1E 6BT, UK; ${ }^{2}$ Centre for Population Health Sciences, Medical School, University of Edinburgh, Edinburgh, UK

Salisbury argues against mandatory vaccination in the UK and suggests that compulsion is not necessary if immunisation coverage is above a "sufficiently high" level.

In 2010 we published a comparison of disease control systems, including childhood vaccination, between Scotland and Hungary. ${ }^{2}$ In 2007, vaccination coverage rates for measles, mumps, and rubella (MMR) and diphtheria, tetanus, and pertussis (DTPa) were essentially $100 \%$ in Hungary, mainly due to longstanding mandatory immunisation. In Scotland, coverage rates of MMR and DTPa were 88.9-93.8\% and $94.1-97.8 \%$, respectively. These rates are reasonably high for a country with voluntary immunisation, and surely above the "sufficiently high" level. Despite this relatively small gap in vaccination coverage, disease rates differed considerably between the two countries.

In 2007 the number of reported pertussis cases was 48 in Hungary and 98 in Scotland ( $0.5 v 1.9$ per 100000 inhabitants). There were no notifications of rubella and measles, and only 16 cases of mumps in Hungary, whereas the Scottish numbers were 146 cases of rubella (incidence rate per $100000: 2.8$ ), 168 cases of measles (3.3), and 2741 cases of mumps (53.3). Because systems of notification, collection of data, and structures in place were similar, these differences were probably not statistical artefacts.

We therefore believe that mandatory vaccination has considerable advantages over the voluntary system, although we agree that its introduction in the UK would present serious challenges. There are lessons to be learnt from other countries.

Competing interests: None declared.

1 Salisbury DM. Childhood vaccination: should it be mandatory? No. BMJ 2012;344:e2435 (15 May.)

2 Stefler D, Bhopal R. Comparison of the Hungarian and Scottish communicable disease control systems: lessons for a convergent European Community. Public Health 2010;124:167-73.

Cite this as: BMJ 2012;344:e4036

๑ $\odot$ BMJ Publishing Group Ltd 2012 